\title{
SH2 domain-containing inositol 5-phosphatase (SHIP2) inhibition ameliorates high glucose-induced de-novolipogenesis and VLDL production through regulating AMPK/mTOR/SREBP1 pathway and ROS production in HepG2 cells
}

Sattar Gorgani-Firuzjaee ${ }^{1}$, Reza Meshkani ${ }^{1}$,

1- Department of Biochemistry, Faculty of Medicine, Tehran University of Medical Sciences, Tehran, I.R Iran.

Running title: SHIP2 regulates lipid metabolism in HepG2 cells

To whom correspondence should be addressed:

Reza Meshkani,

Department of Biochemistry,

Faculty of Medicine,

Tehran University of Medical Sciences,

Tehran, I.R. Iran.

Tel: +98-21-64432502;

Email: rmeshkani@tums.ac.ir 


\section{Abbreviations:}

AMPK: AMP-activated protein kinase,ACC: Acyl COA Carboxylase, BSA: Bovine Serum Albumin, DHE: Dihydroethidium, DPI: Diphenylene iodonium, ERK: Extracellular signal-regulated kinase,FAS: Fatty Acid Synthase,FBS: Fetal bovine serum,FOXO1: Forkhead box protein O1,GFP: Green fluorescent protein, HDL-C: High density lipoprotein cholesterol,HG: High glucose (33 mM),LDL:Low density lipoprotein,JNK: c-June Nterminal kinase,Ins: insulin,MAPK: Mitogen-activated protein kinase,MTP: Microsomal transfer protein, mTOR: mammalian target of rapamycin,NG: Normal glucose $(5.5 \mathrm{mM})$, p38: p38 mitogen-activated protein kinases,PI3-K: phosphatidyl inositol 3kinase,PIP2: Phosphatidylinositol diphosphate, PIP3: Phosphatidylinositol 3,4,5- trisphosphate,PTEN: Phosphatase and tensin homolog,ROS: Reactive oxygen spices,SREBP1c: Sterol Regulatory Element-Binding Proteins, SHIP2: SH2 domain containing inositol 5phosphatase,T2D: SHIP2-DN: SHIP2 Dominant negative mutant gene,SHIP2-WT: SHIP2 wild type gene, T2D: type 2 diabetes, TG: triglyceride,TSC1/2: tuberous sclerosis 1/2, VLDL: Very low density lipoprotein 


\begin{abstract}
Hepatic de-novo lipogenesis and production of triglyceride rich very low density lipoprotein (VLDL)is increased in the state of insulin resistance, however, the role of a negative regulator of the insulin signaling pathway, the SH2 domain-containing inositol 5-phosphatase (SHIP2) in this process, remains unknown. In the present study, we studied the molecular mechanisms linking SHIP2 expression to metabolic dyslipidemia using overexpression or suppression of SHIP2 gene in HepG2 cells exposed to high glucose (33mM). The results showed that high glucose induced SHIP2 mRNA and protein levels in HepG2 cells.Overexpression of the dominant negative mutant SHIP2 (SHIP2-DN)ameliorated high glucose-induced de-novo lipogenesis and secretion of apoB containing lipoprotein in HepG2 cells, as demonstrated by a reduction in both secreted apoB and MTP expression, and decreased triglyceride levels and the expression of lipogenic genes such as SREBP1c, FAS and ACC. Overexpression of the SHIP2-DN decreased high glucose-induced apoB containing lipoproteins secretion via reduction in ROS generation and JNK phosphorylation and Akt activation. Furthermore, using the specific inhibitor and activator, it was found that the AMPK/mTOR/SREBP1 is the signaling pathway that mediates the effects of SHIP2 modulation on hepatic de-novo lipogenesis. Taken together, these findings suggest that SHIP2 is an important regulator ofhepatic lipogenesis and lipoprotein secretion in insulin resistance state.
\end{abstract}

Keywords: De-novo lipogenesis, VLDL, SH2 domain containing inositol 5-phosphatase (SHIP2), Insulin resistance, hyperglycemia, HepG2, liver 


\section{Introduction}

Dyslipidemiais one of the key risk factors for cardiovascular disease in type 2 diabetes (T2D) [1]. The dyslipidemia is characterized by increased plasma concentration of triglycerides (TG), reduced concentration of high density lipoprotein cholesterol (HDL-C) and anincreased concentration of small dense low density lipoprotein (LDL) cholesterol[2]. Evidence from both animal and human studies suggests that insulin resistance (in adipose and liver tissues) is an important underlying cause of hypertriglyceridemia in subjects with metabolic syndrome and $\mathrm{T} 2 \mathrm{D}[3,4]$.In the liver, the insulin controls lipid metabolism through its cell surface receptor and intracellular mediators such as the phosphatidylinositol 3-kinase (PI3-K) and serine-threonine kinase Akt. It was shown that the insulin inhibits apoB100 secretion via the activation of the PI3-K $[5,6]$. Previous studies have also shown that the insulin signaling through PI3-K inhibits the maturation of VLDL lipoprotein particles by preventing the lipidation of VLDL precursor [7]. In insulin resistance state, hepatic VLDL production is increased. In the setting of insulin resistance, fatty acid flux through the plasma into the liver, increased de novo lipogenesis and decreased apoB degradationpromote VLDL overproduction [4].

Activation of the PI3-K pathway enhances the production of the phosphatidylinositol 3,4,5- trisphosphate (PI(3, 4, 5)P3 ) a lipid second messenger molecule that mediates many cellular responses to insulin [8]. $\mathrm{PI}(3,4,5) \mathrm{P} 3$ level is controlled by the activity of PI3-K and lipid phosphatases such as phosphatase and tensin homolog (PTEN) and SH2 domain containing inositol 5-phosphatase (SHIP2) [7, 9-11].SHIP2 hydrolyzes the PI(3, 4, 5)P3 to produce $\mathrm{PI}(3,4) \mathrm{P} 2$. SHIP2 acts as a negative regulation of the insulin signaling both in vitro and in vivo $[12,13]$.An increased expression of SHIP2 has been reported from the skeletal muscle and adipose tissues of $\mathrm{db} / \mathrm{db}$ mice $[14,15]$. It has been demonstrated that SHIP2 overexpression inhibits theinsulin-induced Akt activation, glucose uptake, and glycogen synthesis in 3T3-L1 adipocytes, L6 myotubes[16]andtissues of animal models [17, 18]. In addition,liver-specific inhibition of SHIP2 improvesthe insulin signaling inmice[17, 19].Furthermore, the heterozygous knockout mice of SHIP2 gene were resistant againsthigh-fat diet-induced obesity and insulin resistance [13]. In this models, theinsulin-induced Akt phosphorylation was enhanced in the liver and skeletal muscle, and surprisingly blood TG concentrations and non-esterified free fatty acids were decreased even when they were fed a normal chow diet $[13,20]$. Taken together,these studies demonstrate that the alterations of SHIP2 expression and/or enzymatic function appear to have a profound impact on the development of insulin resistance. However, the function of SHIP2 gene in hepatocytes lipid and lipoprotein metabolism in insulin resistance state remains unknown. In this study we investigated the role of SHIP2 in hepatic de-novo lipogenesis and the secretion of apoB100 containing lipoproteins by modulating its expression in HepG2 cells exposed to high glucose concentration. Our data clearly showed that the modulation of SHIP2 in HepG2 cells could affect lipogenesis and lipoprotein secretion by different mechanisms including the ROS production, JNK activation, and the AMPK/mTO/SREBP1c axis modulation. 


\section{Materials and methods}

\section{Materials}

Dulbecco's modified Eagle's medium (DMEM), fetal bovine serum (FBS), and trypsin EDTA were purchased from Life Technologies(Life Technologies, Gaithersburg, USA). Tissue culture flasks and disposable plastic ware purchased from Greiner Bio-One (Frickenhausen, Germany). Phenyl methyl sulfonyl fluoride (PMSF), protease inhibitor cocktail, and NonidetP-40 were from Roche (Mannheim,Germany). Polyvinylidene difluoride(PVDF) membrane was from Millipore (Schwal-bach, Germany). ECL reagents were from Amersham Pharmacia Corp. (Piscataway, NJ, USA). DHE and MitoSOX red were from Invitrogen (San Diego, CA, USA),Polybrene, puromycin, rapamycin, and metformin were from Sigma.All other reagents and chemicals were from Sigma Aldrich (Taufkirchen,Germany).

\section{Cell culture}

HepG2 and HEK T293 packaging cells were purchased from the Pasteur Institute of Iran. Cells were maintained at $37{ }^{\circ} \mathrm{C}$ (in an atmosphere of 5\% CO2) in DMEM containing 10\% FBS, $2 \mathrm{mM}$ glutamine, and 1\%penicillin-streptomycin. In order to study lipid and lipoprotein metabolism in an insulin resistance state, HepG2 cells were treated with $33 \mathrm{mM}$ D-glucose. D-mannitol was used as an osmotic control. To study the role of the specific signaling pathways, HepG2 cells were pretreated with the appropriate activators (anisomycin, metformin) or inhibitors (MAPKs inhibitors, rapamycin, rotenone, diphenyleneiodonium (DPI) and ascorbic acid)for $2 \mathrm{~h}$.

\section{Retroviral infection}

In order to generate the retrovirus, the transfer and packaging (PMD2 and PUMVC) plasmids were transfected into T293 packaging cells. The plasmids, pBABE- SHIP2 (SHIP2-WT), and pBABE- dominant negative mutant (SHIP2-DN) which was mutated at the catalytic site of the enzyme, were kindly gifted by Dr. Kristopher Erneux [21]. Viral supernatants were collected during 48-96 h after transfection and filtered on 0.44 $\mu \mathrm{m}$ filters. HepG2 cells were infected with the viral supernatants in the presence of $10 \mu \mathrm{g} / \mathrm{ml}$ polybrene. Infected cells were selectively stabled with $2 \mu \mathrm{g} / \mathrm{ml}$ puromycin.

\section{Western blot analysis}

Cell lysate was prepared by homogenization in modified RIPA buffer (50 mM Tris-HCl, pH 7.4, 1\% Triton X-

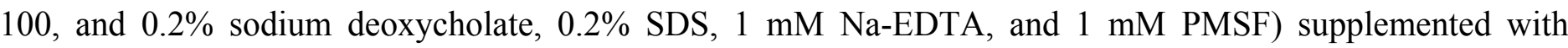
protease inhibitor cocktail. For detection of phosphoproteins, a buffer consisting of $50 \mathrm{mM}$ HEPES, pH 7.5, $150 \mathrm{mM} \mathrm{NaCl}, 100 \mathrm{mM} \mathrm{NaF}, 10 \mathrm{mM}$ EDTA, 10mM Na4P2O7, and 2mM NaVO4, and protease inhibitor cocktail was used. Afterdetermining protein concentrations, equal amounts of protein were subjected to SDS-PAGE, followed by transfer onto PVDF membrane. Blocking was carried out through $2 \mathrm{~h}$ incubation at room 
temperature with 5\% nonfat dry milk or BSA for p-proteins in TBSwith $0.5 \%$ Tween 20 . Blots wereincubated overnight with antibodies against SHIP2 (R\&D system), p-Akt (S473), Akt, SREBP1c, p-ACC, p-FOXO1, pAMPK, p-mTOR, mTOR, p-JNK, p-ERK, p-P38, JNK and AMPK (Cell Signaling Technology, Beverly, MA, USA) and $\beta$-actin (Abcam, Cambridge, MA, USA) at $4{ }^{\circ} \mathrm{C}$. The bandsafter incubating with second HRPconjugated antibodieswere visualized using anenhanced chemiluminescent substrate (ECL). Each experiment was performed at least three times.

\section{Real-Time-PCR}

After treatments, the cells were harvested and total RNA was extracted using RNeasy mini kit. Total RNA was reverse transcribed using the MMulv reverse transcriptase and random hexamer primer (Takara Tokyo, Japan). Gene expression was quantified using specific primers for ACC, FAS, SREBP1c, SHIP2 and MTP with SYBR Green PCR Master Mix (Takara Tokyo, Japan). The sequences of the primers used in the study are in Table 1, supplementary file. The levels of target genes transcript were normalized relative to $\beta$-actin. The amplification protocol for 40 cycles was as follows: $10 \mathrm{~s}$ at $95{ }^{\circ} \mathrm{C}$ for initial activation, $5 \mathrm{~s}$ at $95{ }^{\circ} \mathrm{C}$ for denaturation, and $20 \mathrm{~s}$ at $60{ }^{\circ} \mathrm{C}$ for annealing/extension.

\section{Oil red $\mathrm{O}$ staining}

After $24 \mathrm{~h}$ treatment, the cells were washed in cold PBS and then fixed with 4\% paraformaldehyde solution for $1 \mathrm{~h}$ at room temperature. The cells were washed twice with distilled water and incubated 5 min in $60 \%$ isopropanol. Cells were then stained for $20 \mathrm{~min}$ with fresh oil red $\mathrm{O}$ working solution $(300 \mathrm{mg}$ of oil red $\mathrm{O}$ powder and adding this to $100 \mathrm{ml}$ of $99 \%$ isopropanol). Stained cells were washed thoroughly with distilled water prior to microscopic observation. Images were captured using an Olympus camera mounted on an Olympus upright microscope. In order to quantify, $250 \mu \mathrm{DMSO}$ were added to the dried plates and then the optical density was measured in $510 \mathrm{~nm}$. The results were normalized against total protein.

\section{Determination of triglyceride}

Intra and extra cellular triglycerides content were measured with the Biovision triglyceride quantification Colorimetric/Fluorimetric kit.

\section{Determination of ApoB}

Secreted apoB in cells supernatant was analyzed using the apolipoprotein B human Elisa kit (Abcam, USA) according to the manufacturer's instructions. The data were normalized to intracellular protein levels. Protein concentrations were determined by a BCA protein assay kit.

\section{Reactive oxygen species (ROS) determination}

Intracellular production of ROS was measured using the cell-permeable fluorescent dyes, $\mathrm{CM}-\mathrm{H}_{2} \mathrm{DCFDA}$, dihydroethidium (DHE), and MitoSOX red. CM- $\mathrm{H}_{2}$ DCFDA was applied to monitor intracellular $\mathrm{H}_{2} \mathrm{O}_{2}$ and 
hydroxyl radical production. DHE is oxidized by cytosolic superoxide anion to red fluorescent ethidium, while MitoSOX red is selectively oxidized by superoxide anion in the mitochondria. Briefly, HepG2 ells were grown to reach $50-75 \%$ confluences in 6-well plates. The cells were then washed twice with PBS and loaded with $\mathrm{CM}-\mathrm{H}_{2} \mathrm{DCFDA}(5 \mu \mathrm{M})$ for $30 \mathrm{~min}$ in the dark at $37^{\circ} \mathrm{C}$. Then, cells were washed twice with sterile PBS, resuspended in PBS and analyzed for fluorescence distribution using FACSCaliburflow cytometer. The cells were also plated in 96-well culture dishes. After treatment and then washing twice with PBS, the cells were incubated with DHE $(2 \mu \mathrm{M})$ for $30 \mathrm{~min}$, and MitoSOX red $(5 \mu \mathrm{M})$ for $10 \mathrm{~min}$ in the dark at $37^{\circ} \mathrm{C}$. The fluorescent intensity was then determined on a fluorescent plate reader.

\section{Statistical analyses}

All statistical analyses were performed using SPSS18.0. (SPSS, Chicago, IL, USA). Comparisons among all groups were performed with the one-way analysis of variance. If statistical significance was found, the Tukey posthoc test was performed. Values of $\mathrm{p}<0.05$ were considered statistically significant. Results are expressed as the mean \pm SEM of at least three independent experiments.

\section{Results}

\section{Importance of SHIP2 inhibition in high glucose-induced lipogenesis}

Dyslipidemia is one of the important events in hyperglycemic state such as T2D [22, 23]. To show the role of SHIP2 in high glucose-induced de-novo lipogenesis and lipoprotein secretion, wefirst assessed SHIP2 expression in HepG2 cells treated with 33mM glucose. High glucoseenhanced SHIP2 expression both at mRNA and protein levelsmore than 90\% $(\mathrm{p}<0.001)$ (Fig 1A-B).To verifythe role of SHIP2 in high glucose-induced lipogenesis, we first generated the stable HepG2 cells overexpressing or suppressing the activity of SHIP2. These cells had 3.4 and 3.7 fold more SHIP2 protein level of the dominant negative (SHIP2-DN) and wild-type (SHIP2-WT), respectively, compared to the GFP control cells (Fig 2A). In order to confirm that our retrovirus systems carrying the SHIP2 genes work efficiently, the phosphorylation of Akt, a downstream protein of SHIP2 was evaluated. As shown in Fig 2B, overexpression of the SHIP2-DN and SHIP2-WT genes result in 1.66 and 0.52 fold changes in Akt phosphorylation, respectively, confirming the functionality of SHIP2 in stable HepG2 cells.

Wethen evaluated different factors related to lipid and lipoprotein metabolism in SHIP2-DN cells. Inhibiting the activity of SHIP2 inSHIP2-DNcells reducedtotal lipid content induced by high glucose(Fig 2C-D). Further experiments demonstrated that both intra and extracellular TG content significantly reduced in SHIP2-DN cells treated with high glucose (27 and 34\%, respectively)(Fig 2 E-F). The amount of secreted apoB was also affected by SHIP2 modulation, as the SHIP2-DN cells treated with high glucose had lower apoB levels compared to control cells (Fig 2G). We confirmed above findings by investigating the expression of importantgenes involved 
inlipid and lipoprotein metabolism such as MTP, ACC, FAS, and SREBP1c. The expression of all these genes significantly diminished in SHIP2-DN cells treated with high glucose (Fig $2 \mathrm{H}$ and I, and supplementary file, Fig 1). Our data from western blot analysis also revealed that the inhibition of SHIP2 in SHIP2-DN cells could significantly attenuate high glucose-induced SREBP1c expression (Fig 2L). Furthermore, theinhibition of SHIP2 could prevent high glucose-reduced ACC phosphorylation in hepatocytes (Fig 2K). We finally studiedthe FOXO1 phosphorylation in SHIP2-DNcells. As shown in Figure 1M,SHIP2-DN cells obviously had more than 2 fold FOXO1 phosphorylation.On the other hand, overexpression of SHIP2-WT led to a significant increase in both secretion of apoB containing lipoproteins and de-novo lipogenesis, as demonstrated by an enhance in both secreted apoB and MTP expression, increased intra and extracellular TG levels and enhanced the expression of lipogenic genes such as SREBP1c, FAS and ACC. Totally, above findings demonstrate that SHIP2 inhibition ameliorates high glucose-induced lipogenesis and secretion of apoB lipoproteinsin HepG2 cells.

\section{Effect of SHIP2 inhibition on high glucose-induced ROS production}

It has been previously shown that high glucose induces oxidative stress in different cells [24, 25]. To investigate the mechanismby which SHIP2 inhibition could ameliorate high glucose-induced lipogenesis in HepG2 cells,we hypothesized that the effects of SHIP2 on lipid and lipoprotein metabolism might be mediated throughthe ROSproduction. To evaluate this hypothesis, total lipid content and the expression of lipogenic genes were analyzed in HepG2 cells treated with high glucose and SHIP2-WTcells at the presence of $50 \mu \mathrm{M}$ ascorbic acid as a ROS scavenger. Ascorbic acid couldsignificantly reduce lipid content in both HepG2 cells treated with high glucose and SHIP2-WT cells (Fig 3A). Similarly, ascorbic acidcould prevent increased apoB secretion and the expression of MTP, SREBP1c, ACC and FAS in HepG2 cells treated with high glucose and the SHIP2-WTcells as well (Fig 3B-F).

In order to demonstrate the direct role of ROS in de novo lipogenesis, ROS production was measuredby using the fluorescent probesCM-H ${ }_{2}$ DCFDA and DHE. As shown in Fig 3G and Fig 2 of the supplementary file, the SHIP2-DNcells treated with high glucose had significantly lower ROS production assessed by both CM$\mathrm{H}_{2}$ DCFDA and DHE probes, whereas theSHIP2-WTcells had an increasedROS production compared to their controls. To demonstrate the source of ROS induced by high glucose and SHIP2 modulation in hepatocytes, we examined the cellular pathway involved in ROS production, including NADPH oxidase and mitochondria mediated pathways. We first treated the cells with different concentrations of diphenylene iodonium (DPI), an inhibitor of NADPH oxidase and rotenone, an inhibitor of mitochondrial respiratory chain complex I. Our results showed that $5 \mu \mathrm{M}$ DPI and $0.1 \mu \mathrm{M}$ rotenone have the highest capacity to attenuate total lipid induction in highglucose treated and SHIP2-WT cells (Fig $3 \mathrm{I}$ and K). We confirmed this data by measurement of TG concentrations (Fig $3 \mathrm{~L}$ and $\mathrm{M}$ ). We also measured mitochondrial ROS level using MitoSOX Red, a 
mitochondrial superoxide indicator. As shown in Fig 3H, high glucose and overexpression of SHIP2-WT led to a significant increase in mitochondrial ROS level compared to controls. Taken together, these results suggest that both NADPH oxidase and mitochondria constitute the major source of enhanced oxidative stress in high glucose-treated and SHIP2-WT cells.

\section{The role of MAPK signaling pathway in SHIP2 mediated de novo lipogenesis}

To address the signaling pathway which link SHIP2 to lipid and lipoprotein metabolism, we targeted Mitogen-activated protein kinase (MAPK) pathway. We first pretreated the cells with MAPKs inhibitors

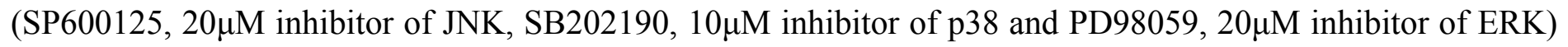
and then oil red o staining and real-time PCR for gene expression analyses were performed. The results showed that only JNK inhibitor significantly reduced total lipid content, apoB secretion and the expression of MTP,SREBP1c, ACC and FAS genes in HepG2 cells treated with high glucose and also SHIP2-WTcells compared to control cells (Fig 4A-C). We confirmed this finding by detecting the phosphorylation of the MAPKs proteins. Our results showed that only JNK phosphorylation significantly increased at the presence of high glucose in HepG2 cells (Fig 4D). Interestingly, SHIP2 modulation affects JNK phosphorylation, as the inhibition of SHIP2 in SHIP2-DN cells reduced JNK phosphorylation by $52 \%$, whereas overexpressing SHIP2WT led to $65 \%$ more phosphorylation of JNK in HepG2 cells (Fig 4D). To confirm these data, HepG2 cells were pretreated with anisomysin, a JNK activator. As shown in Fig 4E, anisomysin significantly induced the phosphorylation of JNK. Importantly, anisomysin significantly induced total lipid content in HepG2 cells treated with both normal and high glucose (Fig 4F). While total lipid content and apoB secretionreduced in SHIP2-DN cells treated with high glucose, adding anisomysin to the media prevented the effect of SHIP2 inhibition on these factors (Fig 4G). In addition, as shown in Fig 4H and Fig 3of the supplementary file, the expression of MTP, ACC, FAS and SREBP1c significantly were induced by anisomysin in HepG2 cells.Furthermore, anisomysin could neutralize the effect of SHIP2 inhibition on high glucose-induced lipogenesis and secretion of apoB lipoproteinsin HepG2 cells.Taken together, these data suggest that high glucose induces both lipogenesis and secretion of apoB lipoproteinsby a mechanism that involves the activation of JNK in HepG2 cells. It also appears that the effects of SHIP2 on lipid and lipoprotein metabolism might be exerted through the induction of JNK phosphorylation in hepatocytes.

It has been previously shown that JNK is activated in response to ROS production[26]. To find the link between JNK activation and ROS production, we treated the cells at the presence of high glucose and ascorbic acid. High glucose could increase JNK phosphorylation by 2.2 fold, whereas ascorbic acid reversed the effect of high glucose on JNK phosphorylation. Interestingly, ascorbic acid also reduced JNK phosphorylation in SHIP2- 
WT cells, suggesting the existence of a link between ROS production and JNK activation in SHIP2 modulated cells (Fig 4I).

Previous studies have shown a link between JNK and Akt. It has been reported that JNK activation leads to a reduction in Akt phosphorylation [26]. Accordingly, we hypothesized that the effects of SHIP2 modulation on lipid and lipoprotein metabolism might be exerted through this pathway. We first investigated the link between JNK and Akt phosphorylation. Using a specific inhibitor of JNK, we found that the inhibition of JNK leads to increased Akt phosphorylation in HepG2 cells treated with high glucose and the SHIP2-WT cells (Fig 4K). On the other hand, anisomysin decreased Akt phosphorylation in both the HepG2 and SHIP2-DN cells treated with high glucose (Fig 4L). Regarding the effects of SHIP2 modulation on Akt phosphorylation, we found that the inhibiting SHIP2 in SHIP2-DN cells could ameliorate high glucose-reduced Akt phosphorylation, whereas overexpression of SHIP2-WT led to decreasedphosphorylation of Akt in HepG2 cells (Fig 4M). Altogether, these findings suggest that the effects of SHIP2 on lipid metabolism might be mediated by a mechanism involving the activation of ROS/JNK followed by the inhibition of Akt activity in hepatocytes.

\section{Effect SHIP2 Inhibition on AMPK-mTOR-SREBP1c pathway in high glucose condition}

Studies on hepatic lipogenesis have targeted nutrient-dependent activation of the AMPK/mTOR/SREBP1c pathway in hepatic denovo lipogenesis [27-31]. To investigate whether SHIP2 modulation influence these pathways, we first evaluated AMPK phosphorylation at the presence of high glucose.High glucose significantlyreduced the phosphorylation of AMPK in HepG2 cells, whereasthe phospho-AMPK levels wereincreased and decreased in SHIP2-DN cells treated with high glucose and the SHIP2-WT cells, respectively (Fig 5A). We then assessed the mTOR ser 2481 phosphorylation level in SHIP2 modulated cells. As shown in Fig 5B,high glucose inducedthe phosphorylation of mTOR 3.1 fold.Inhibiting SHIP2 in SHIP2DN cells reduced mTOR phosphorylation by 30\% and overexpression of SHIP2-WTinduced phospho-mTOR level 2.2 fold versus the GFP control cells. To confirm the role of this pathway in lipogenesis, we treated the cells with high glucose and rapamycin as mTOR inhibitor.Rapamycin significantly reducedtotal lipid content,TG levels and SREBP1c protein levelin the cells suggesting the importance of mTOR in hepatic lipogenesis (Fig 5D-E). To identify the role of AMPK in lipogenesis, we treated the cells with metformin as an activator of AMPK. Interestingly metformin was able to decrease lipogenesis in HepG2 cells, as demonstrated by changes in total lipid content, TG level, SREBP1c protein and ACC phosphorylation levels(Fig 5C, F). To study whether AMPK is the upstream of mTOR or vice versa, we measured phospho-AMPK and phosphomTOR levels in the cells treated with rapamycin and metformin. Our resultssuggested that AMPK activates mTOR pathway. In this regard rapamycin did not significantly change the AMPK phosphorylation, (Fig 
5G), while, metformin was able to significantly reduce high glucose-induced mTOR phosphorylation in HepG2 cells (Fig 5H).

We further focused on whether the effect of SHIP2 on hepatic lipogenesis is also mediated through the AMPK/mTOR pathway. To this aim we analyzed SREBP1c and phospho-ACC levelsat the presence of metformin and rapamycin in SHIP2-WT cells. The results showed that rapamycin reducedSREBP1c protein and increasedACC phosphorylation levelsin the SHIP2-WTcells (Fig 5G). Furthermore, metformin treatment resulted in a lowerlevel of SREBP1c protein and a higher level of phospho-ACC in the SHIP2-WT cells (Fig $5 \mathrm{H})$.Altogether,these data demonstrate that SHIP2overexpression enhances lipogenesis in HepG2 cells via the activation of AMPK/mTOR/SREBP1c pathway.

\section{Discussion}

Hypertriglyceridemia a pathological feature of the metabolic syndrome and T2D, is characterized by augmented production and/or retarded clearance of TG-rich particles, such as VLDL [32, 33]. While the pathophysiology of hypertriglyceridemia is poorly understood, its close association with visceral adiposity and T2D implicates insulin resistance as a causative factor for hypertriglyceridemia [34]. VLDL production in the liver largely depends on substrate availability and sensitivity of the cells to the insulin. [35]. In T2D and metabolic syndrome, the aberrant insulin action in combination with increased influx of FFAs into the liver promotes excessive VLDL-TG production, contributing to the pathogenesis of hypertriglyceridemia [36]. In spite of many efforts, the underlying molecular mechanism of hepatic VLDL-TG overproduction and key players in this process is a matter of debate. In the present study we focused on SHIP2, an intermediate of the insulin signaling, and investigated its role in hepatic lipogenesis and secretion of apoB containing lipoproteins in a high glucose condition.

Many complications of chronic metabolic diseases such as metabolic syndrome and T2D can be attributed to hyperglycemia. One of the consequences of elevated glucose level is hepatic lipid accumulation and increase of VLDL secretion [37,38]. Despite many studies, the molecular mechanism of hyperglycemia-induced lipogenesis is not well understood. The present data suggest that the effects of high glucose on lipid and lipoprotein metabolism may at least in part be mediated by SHIP2.High glucose could induce SHIP2 expression at mRNA and protein levels in HepG2 cells. It is worthy note that an increased expression of SHIP2 in the state of insulin resistance has been previously reported [15, 39, 40]. Furthermore, SHIP2 inhibition via the dominant negative expression significantly prevented hyperglycemia-induced de-novo lipogenesis as demonstrated by a reduced the expression of key lipogenic genes such as FAS, ACC and transcription factors including the SREBP1c, FOXO1. SHIP2 inhibition also decreased secretion of apoB containing lipoproteins. These findings suggest that the inhibition of SHIP2 via reduction of lipid (TG) and protein content of VLDL (apoB) and 
suppressing the responsible protein in VLDL assembly (MTP) leads to decreasing VLDL production in high glucose condition. These observations suggest that a reduction in SHIP2 expression may prevent carbohydrateinduced changes in insulin sensitivity and the associated abnormalities in lipid and lipoprotein metabolism.

The mechanism by which SHIP2influence lipid and lipoprotein metabolism is most likely regulated indirectly via the changes in insulin sensitivity. Insulin is an important regulator of lipid and lipoprotein metabolism, and hepatic lipogenesis and lipoprotein production are tightly controlled by the insulin action [41]. Insulin induces SREBP-1c expression by the PI3-K/Akt-dependent mechanism, but the downstream effectors are not clearly understood [42].Insulin has also been shown to block apoB secretion by a mechanism involving the degradation of intracellular apoB [43], and it is thought that this effect can be blocked with the induction of hepatic insulin resistance. Previous studies have also reported that the insulin-suppressive effects on apoB secretion need PI3-K activation. Activation of PI3-K was thought to mediate the inhibition of apoB secretion possibly via the generation of the PI(3, 4, 5)P3[44]. In the present study SHIP2 suppression increases thePI3K/Akt activity and therefore it is plausible to suggest that SHIP2 inhibition may promote the suppressive effect of insulin on apoB secretion by activating the PI3-K/Akt pathway. In addition, our data demonstrated that high glucose alone and SHIP2 overexpression despite of a reduction in Akt activation leads to an increased the expression of SREBP1c and its target genes, suggesting an Akt-independent pathway operating the lipogenic effect of high glucose and SHIP2 overexpression in HepG2 cells. Thus, it appears that SHIP2 can potentially affect the rate of de-novo lipogenesis, resulting in alterations of the secretory pool of lipid substrates available for VLDL assembly and secretion. In accordance with this notion, it has been reported that liver-specific inhibition of SHIP2 improve glucose tolerance in diabetic KKA mice by affecting glucose metabolism [17]. But this study, however, did not examine the alterations of lipid and lipoprotein metabolism associated with the inhibition of SHIP2 expression. Our finding appears to extend these observations and further suggest that reducing SHIP2 expression can significantly attenuate hepatic de-novo lipogenesis and lipoprotein secretion most likely as a result of enhanced hepatic insulin signaling.

Another mechanism linking SHIP2 to lipid metabolism is oxidative stress. In vitro and in vivo studies have demonstrated a correlation between the oxidative stress and lipid accumulation in liver tissue [45, 46].However, the direct molecular mechanism linking the oxidative stress to enhanced lipogenesis remains unknown. A growing body of evidence suggests that the oxidative stress due to excessive production of ROS is the main contributor to the pathophysiology of diabetic complications under the hyperglycemic conditions[47, 48].However, the exact source, and cellular location of the glucose-induced ROS is still unclear. Both mitochondria and NADPH oxidase have been suggested to be the major sources of ROS production in different cells treated with glucose[49, 50]. In the present study, we used DPI, an inhibitor of NADPH oxidase and 
rotenone an inhibitor of mitochondrial respiratory chain complex I to explore the importance of mitochondria versus NADPH oxidase derived ROS in high glucose-induced lipogenesis of HepG2 cells. Our observations suggested that both mitochondria and NADPH oxidase are the sources of ROS in high glucose-induced lipogenesis of Hepatocytes. Consistent with our results, DPI was found to decrease ROS production by NADPH oxidase in HepG2 cells treated with high glucose concentration [51]. Our data also revealed that both mitochondria and NADPH oxidase are involved in lipogenesis induced by overexpression of SHIP2-WT in HepG2 cells.

The present data is first to suggest that high glucose-inducedde-novo lipogenesis is mediated through ROSdependent JNK activation. In this regard, a ROS scavenger and a JNK inhibitor prevented high glucose-induced de-novo lipogenesis and secretion of apoB containinglipoproteins. JNK activation then leads to inactivation of Akt in HepG2 cells. Inactivation of Akt, as stated earlier, mediates the induction of apoB secretion and VLDL overproduction [44]. Interestingly, SHIP2 modulation was able to affect the status of the oxidative stress and JNK activation in HepG2 cells.SHIP2 overexpression enhanced ROS production and JNK activation, whereas overexpression of the SHIP2-DN gene led to a reduction in ROS production and JNK phosphorylation in the cells treated with high glucose. These observations highlight that alterations in the oxidative status and JNK activation might be the link between high glucose, SHIP2 modulation and lipogenesis and VLDL production in HepG2 cells. In the present study we did not focus on the link between SHIP2 and JNK activation, however, it is possible that the effect of SHIP2 on JNK activation is mediated through its partner, JNK-interacting protein 1 (JIP1). SHIP2 positively regulates the tyrosine phosphorylation of JIP1. It has been previously reported that SHIP2 has a positive influence on mixed-lineage kinase3 (MLK3)/JIP1-induced JNK1 activity[52].

It is worth also noting that the effects of ROS/JNK pathway on de-novo lipogenesis is Akt-independent in both the SHIP2 modulated and HepG2 high glucose treated cells. While SREBP1cinduction is usually dependent onthe activation of Akt, we observed a decrease of Akt phosphorylation byJNK activation in SHIP2WT and HepG2 cells treated with high glucose. However, it appears that the effect of ROS/JNK pathway on VLDL production occurs through an Ak-dependent mechanism.

The mechanistic target of rapamycin (mTOR) is a serine/threonine protein kinase that senses the nutrients and growth factors and regulates several biological processes required for the promotion of cell growth [53]. Aberrant mTOR signaling is involved in the pathogenesis of several diseases such as obesity, T2D, cancer and neurodegenerative disorders [53]. mTOR is present in two distinct complexes, mTOR complex 1 (mTORC1), which is partially inhibited by rapamycin and mTORC2. Growth factors and hormones (e.g. insulin) activate mTORC1 via the PI3-K/Akt pathway. Akt inactivates the tuberous sclerosis $1 / 2$ (TSC1/2) to prevent the 
inhibition of mTORC1 [54]. Alternatively, low ATP levels lead to AMP-activated protein kinase (AMPK)dependent activation of TSC2 and phosphorylation of raptor to reduce the mTORC1 signaling. It is now clear that mTORC1 promotes hepatic lipogenesis by activating the SREBP1 by inducing the SREBP1 expression, processing, and nuclear accumulation[55]. In this regard, our data support the role of mTORC1 in hepatic lipogenesis, as mTORC1 phosphorylation (ser2481) increased in parallel with the SREBP1c expression in the state of high glucose. However, our results did not demonstrate an Akt-dependent activation of mTORC1 and it seems that a high glucose suppression of phospho-AMPK promotes the mTORC1 activation in HepG2 cells. It is of note that an Akt-independent regulation of mTORC1 by energy-sensing pathways (AMPK) has been mostly found in cancer cells [56]. AMPK communicates the energy status of the cell to the mTOR pathway by both indirect and direct inhibition of mTORC1. AMPK inhibits the mTOR indirectly by phosphorylating the tumor suppressor TSC2 at S1227 and S1345, or by direct phosphorylation on raptor (S722 and S792), a component of mTORC1[57]. Our data also revealed that the effect of SHIP2 on hepatic lipogenesis is mediated by an Akt-independent activation of mTORC1-SREBP1c pathway. While SHIP2-WT overexpression as expected resulted in a decreased Akt phosphorylation, we were not able to observe a parallel inhibition of mTORC1in HepG2 cells. In addition, the inhibition of SHIP2 in SHIP2-DN cells exposed to high glucose caused AMPKactivation leading to a decrease in the activity of mTOR-SREBP1c pathway. This Aktindependent lipogenesis in both high glucose and SHIP2-WT overexpression states appears to be unexpected. One explanation for this unexpected finding can be attributed to the nature of the cell line we used. HepG2 is a human hepatocellular liver carcinoma cell line and the activation of mTORC1 is important for the survival, proliferation and apoptosis resistance of this cell [58]. Furthermore, AMPK is dysfunctional in patients with hepatocellular carcinoma, and low phospho-AMPK staining is correlated with aggressive clinicopathologic features and poor prognosis [59]. Therefore, it appears that the AMPK inhibition accompanied by the mTOR activation is a dominant signaling pathway that might promote the proliferation of HepG2 cells. Further studies in primary hepatocytes might help to have a better conclusion on the role of high glucose and SHIP2 modulation on these pathways.

In summary,our study provides the evidence that SHIP2 regulates the de-novo hepatic lipogenesis and the secretion of apoB containing lipoproteins in HepG2 cells. We discovered that the effects of high glucose on lipid and lipoprotein metabolism are mediated through two mechanisms including the ROS/JNK and theAMPK/mTROC1/SREBP1 pathways. Interestingly, the inhibition of SHIP2 expression could ameliorate high glucose-induced hepatic lipogenesis by reversing the mentioned pathways (Fig 6). According to thesefindings, it appears that the inhibition of SHIP2 could potentially be a target in treatment of dyslipidemia in T2D and metabolic syndrome subjects. However, further in vivo and in vitro studies are needed to elucidate the potential role of SHIP2 in control of hepatic lipogenesis. 


\section{Acknowledges:}

This work was financially supported by grants from the Iran National Science Foundation (93009198) and the deputy of research, Tehran University of Medical Sciences.

\section{References}

[1] Day, C. Metabolic syndrome, or What you will: definitions and epidemiology. Diabetes and Vascular Disease Research 4:32-38; 2007.

[2] Meshkani, R.; Adeli, K. Mechanisms linking the metabolic syndrome and cardiovascular disease: role of hepatic insulin resistance. The Journal of Tehran University Heart Center 4:77-84; 2009.

[3] Grundy, S. M. Atherogenic dyslipidemia associated with metabolic syndrome and insulin resistance. Clinical cornerstone 8:S21-S27; 2006.

[4] Meshkani, R.; Adeli, K. Hepatic insulin resistance, metabolic syndrome and cardiovascular disease. Clinical biochemistry 42:1331-1346; 2009.

[5] Sparks, J.; Phung, T.; Bolognino, M.; Sparks, C. Insulin-mediated inhibition of apolipoprotein B secretion requires an intracellular trafficking event and phosphatidylinositol 3-kinase activation: studies with brefeldin $A$ and wortmannin in primary cultures of rat hepatocytes. Biochem. J 313:567-574; 1996.

[6] Sidiropoulos, K. G.; Meshkani, R.; Avramoglu-Kohen, R.; Adeli, K. Insulin inhibition of apolipoprotein B mRNA translation is mediated via the PI-3 kinase/mTOR signaling cascade but does not involve internal ribosomal entry site (IRES) initiation. Archives of biochemistry and biophysics 465:380-388; 2007.

[7] Brown, A.-M.; Gibbons, G. F. Insulin Inhibits the Maturation Phase of VLDL Assembly via a Phosphoinositide 3Kinase-Mediated Event. Arteriosclerosis, thrombosis, and vascular biology 21:1656-1661; 2001.

[8] Farese, R. V.; Sajan, M. P.; Standaert, M. L. Insulin-sensitive protein kinases (atypical protein kinase C and protein kinase B/Akt): actions and defects in obesity and type II diabetes. Experimental Biology and Medicine 230:593-605; 2005.

[9] Cristofano, A. D.; Pesce, B.; Cordon-Cardo, C.; Pandolfi, P. P. Pten is essential for embryonic development and tumour suppression. Nature genetics 19:348-355; 1998.

[10] Giuriato, S.; Pesesse, X.; Bodin, S.; Sasaki, T.; Viala, C.; Marion, E.; Penninger, J.; Schurmans, S.; Erneux, C.; Payrastre, B. SH2-containing inositol 5-phosphatases 1 and 2 in blood platelets: their interactions and roles in the control of phosphatidylinositol 3, 4, 5-trisphosphate levels. Biochem. J 376:199-207; 2003.

[11] Vinciguerra, M.; Foti, M. PTEN and SHIP2 phosphoinositide phosphatases as negative regulators of insulin signalling. Archives of physiology and biochemistry 112:89-104; 2006.

[12] Ishihara, H.; Sasaoka, T.; Hori, H.; Wada, T.; Hirai, H.; Haruta, T.; Langlois, W. J.; Kobayashi, M. Molecular cloning of rat SH2-containing inositol phosphatase 2 (SHIP2) and its role in the regulation of insulin signaling. Biochemical and biophysical research communications 260:265-272; 1999.

[13] Sleeman, M. W.; Wortley, K. E.; Lai, K.-M. V.; Gowen, L. C.; Kintner, J.; Kline, W. O.; Garcia, K.; Stitt, T. N.; Yancopoulos, G. D.; Wiegand, S. J. Absence of the lipid phosphatase SHIP2 confers resistance to dietary obesity. Nature medicine 11:199-205; 2005.

[14] Hori, H.; Sasaoka, T.; Ishihara, H.; Wada, T.; Murakami, S.; Ishiki, M.; Kobayashi, M. Association of SH2-containing inositol phosphatase 2 with the insulin resistance of diabetic db/db mice. Diabetes 51:2387-2394; 2002.

[15] Gorgani-Firuzjaee, S.; Ahmadi, S.; Meshkani, R. Palmitate induces SHIP2 expression via the ceramide-mediated activation of NF-KB, and JNK in skeletal muscle cells. Biochemical and biophysical research communications 450:494-499; 2014.

[16] Wada, T.; Sasaoka, T.; Funaki, M.; Hori, H.; Murakami, S.; Ishiki, M.; Haruta, T.; Asano, T.; Ogawa, W.; Ishihara, H. Overexpression of $\mathrm{SH} 2$-containing inositol phosphatase 2 results in negative regulation of insulin-induced metabolic actions in 3T3-L1 adipocytes via its 5'-phosphatase catalytic activity. Molecular and cellular biology 21:1633-1646; 2001. [17] Grempler, R.; Zibrova, D.; Schoelch, C.; van Marle, A.; Rippmann, J. F.; Redemann, N. Normalization of Prandial Blood Glucose and Improvement of Glucose Tolerance by Liver-Specific Inhibition of SH2 Domain-Containing Inositol 
Phosphatase 2 (SHIP2) in Diabetic KKAy Mice SHIP2 Inhibition Causes Insulin-Mimetic Effects on Glycogen Metabolism, Gluconeogenesis, and Glycolysis. Diabetes 56:2235-2241; 2007.

[18] Kagawa, S.; Soeda, Y.; Ishihara, H.; Oya, T.; Sasahara, M.; Yaguchi, S.; Oshita, R.; Wada, T.; Tsuneki, H.; Sasaoka, $\mathrm{T}$. Impact of transgenic overexpression of $\mathrm{SH} 2$-containing inositol $5^{\prime}$-phosphatase 2 on glucose metabolism and insulin signaling in mice. Endocrinology 149:642-650; 2008.

[19] Cabaro, S.; Liotti, A.; Longo, M.; Parrillo, L.; Pagano, T. B.; Raciti, G. A.; Penkov, D.; Paciello, O.; Miele, C.; Formisano, P. PREP1 deficiency downregulates hepatic lipogenesis and attenuates steatohepatitis in mice. Diabetologia 56:2713-2722; 2013.

[20] Kagawa, S.; Sasaoka, T.; Yaguchi, S.; Ishihara, H.; Tsuneki, H.; Murakami, S.; Fukui, K.; Wada, T.; Kobayashi, S.; Kimura, I. Impact of SRC homology 2-containing inositol 5'-phosphatase 2 gene polymorphisms detected in a Japanese population on insulin signaling. The Journal of Clinical Endocrinology \& Metabolism 90:2911-2919; 2005.

[21] Zhang, J.; Liu, Z.; Rasschaert, J.; Blero, D.; Deneubourg, L.; Schurmans, S.; Erneux, C.; Pesesse, X. SHIP2 controls Ptdlns $(3,4,5)$ P 3 levels and PKB activity in response to oxidative stress. Cellular signalling 19:2194-2200; 2007.

[22] Aguirre, L.; Portillo, M. P.; Hijona, E.; Bujanda, L. Effects of resveratrol and other polyphenols in hepatic steatosis. World journal of gastroenterology: WJG 20:7366; 2014.

[23] Kim, J. J.; Tan, Y.; Xiao, L.; Sun, Y.-L.; Qu, X. Green tea polyphenol epigallocatechin-3-gallate enhance glycogen synthesis and inhibit lipogenesis in hepatocytes. BioMed research international 2013; 2013.

[24] Chandrasekaran, K.; Swaminathan, K.; Chatterjee, S.; Dey, A. Apoptosis in HepG2 cells exposed to high glucose. Toxicology in Vitro 24:387-396; 2010.

[25] Chen, J.-Y.; Chou, H.-C.; Chen, Y.-H.; Chan, H.-L. High glucose-induced proteome alterations in hepatocytes and its possible relevance to diabetic liver disease. The Journal of nutritional biochemistry 24:1889-1910; 2013.

[26] Taheripak, G.; Bakhtiyari, S.; Rajabibazl, M.; Pasalar, P.; Meshkani, R. Protein tyrosine phosphatase 1B inhibition ameliorates palmitate-induced mitochondrial dysfunction and apoptosis in skeletal muscle cells. Free Radical Biology and Medicine 65:1435-1446; 2013.

[27] Kim, K.; Pyo, S.; Um, S. H. S6 kinase 2 deficiency enhances ketone body production and increases peroxisome proliferator-activated receptor alpha activity in the liver. Hepatology 55:1727-1737; 2012.

[28] Laplante, M.; Sabatini, D. M. An emerging role of mTOR in lipid biosynthesis. Current Biology 19:R1046-R1052; 2009.

[29] Larter, C. Z.; Farrell, G. C. Insulin resistance, adiponectin, cytokines in NASH: Which is the best target to treat? Journal of hepatology 44:253-261; 2006.

[30] Mihaylova, M. M.; Shaw, R. J. The AMPK signalling pathway coordinates cell growth, autophagy and metabolism. Nature cell biology 13:1016-1023; 2011.

[31] Nelson, L. E.; Valentine, R. J.; Cacicedo, J. M.; Gauthier, M.-S.; Ido, Y.; Ruderman, N. B. A novel inverse relationship between metformin-triggered AMPK-SIRT1 signaling and p53 protein abundance in high glucose-exposed HepG2 cells. American Journal of Physiology-Cell Physiology 303:C4-C13; 2012.

[32] Ginsberg, H. N. Treatment for patients with the metabolic syndrome. The American journal of cardiology 91:2939; 2003.

[33] Moller, D. E.; Kaufman, K. D. Metabolic syndrome: a clinical and molecular perspective. Annu. Rev. Med. 56:4562; 2005.

[34] Avramoglu, R. K.; Basciano, H.; Adeli, K. Lipid and lipoprotein dysregulation in insulin resistant states. Clinica Chimica Acta 368:1-19; 2006.

[35] den Boer, M. A.; Voshol, P. J.; Kuipers, F.; Romijn, J. A.; Havekes, L. M. Hepatic glucose production is more sensitive to insulin-mediated inhibition than hepatic VLDL-triglyceride production. American Journal of PhysiologyEndocrinology and Metabolism 291:E1360-E1364; 2006.

[36] Kamagate, A.; Qu, S.; Perdomo, G.; Su, D.; Kim, D. H.; Slusher, S.; Meseck, M.; Dong, H. H. FoxO1 mediates insulin-dependent regulation of hepatic VLDL production in mice. The Journal of clinical investigation 118:2347-2364; 2008.

[37] Fu, S.; Watkins, S. M.; Hotamisligil, G. S. The role of endoplasmic reticulum in hepatic lipid homeostasis and stress signaling. Cell metabolism 15:623-634; 2012. 
[38] Li, H.; Min, Q.; Ouyang, C.; Lee, J.; He, C.; Zou, M.-H.; Xie, Z. AMPK activation prevents excess nutrient-induced hepatic lipid accumulation by inhibiting mTORC1 signaling and endoplasmic reticulum stress response. Biochimica et Biophysica Acta (BBA)-Molecular Basis of Disease 1842:1844-1854; 2014.

[39] Marion, E.; Kaisaki, P. J.; Pouillon, V.; Gueydan, C.; Levy, J. C.; Bodson, A.; Krzentowski, G.; Daubresse, J.-C.; Mockel, J.; Behrends, J. The gene INPPL1, encoding the lipid phosphatase SHIP2, is a candidate for type 2 diabetes in rat and man. Diabetes 51:2012-2017; 2002.

[40] Stiles, B.; Wang, Y.; Stahl, A.; Bassilian, S.; Lee, W. P.; Kim, Y.-J.; Sherwin, R.; Devaskar, S.; Lesche, R.; Magnuson, M. A. Live-specific deletion of negative regulator Pten results in fatty liver and insulin hypersensitivity. Proceedings of the National Academy of Sciences of the United States of America 101:2082-2087; 2004.

[41] Qiu, W.; Avramoglu, R. K.; Dubé, N.; Chong, T. M.; Naples, M.; Au, C.; Sidiropoulos, K. G.; Lewis, G. F.; Cohn, J. S.; Tremblay, M. L. Hepatic PTP-1B Expression Regulates the Assembly and Secretion of Apolipoprotein B-Containing Lipoproteins Evidence From Protein Tyrosine Phosphatase-1B Overexpression, Knockout, and RNAi Studies. Diabetes 53:3057-3066; 2004.

[42] Ferre, P.; Foufelle, F. Hepatic steatosis: a role for de novo lipogenesis and the transcription factor SREBP-1c. Diabetes, Obesity and Metabolism 12:83-92; 2010.

[43] Sparks, J. D.; Sparks, C. E. Insulin modulation of hepatic synthesis and secretion of apolipoprotein B by rat hepatocytes. Journal of biological chemistry 265:8854-8862; 1990.

[44] Phung, T. L.; Roncone, A.; de Mesy Jensen, K. L.; Sparks, C. E.; Sparks, J. D. Phosphoinositide 3-kinase activity is necessary for insulin-dependent inhibition of apolipoprotein B secretion by rat hepatocytes and localizes to the endoplasmic reticulum. Journal of Biological Chemistry 272:30693-30702; 1997.

[45] Graves, J. A.; Metukuri, M.; Scott, D.; Rothermund, K.; Prochownik, E. V. Regulation of reactive oxygen species homeostasis by peroxiredoxins and c-Myc. Journal of Biological Chemistry 284:6520-6529; 2009.

[46] Kim, Y.-M.; Shin, H.-T.; Seo, Y.-H.; Byun, H.-O.; Yoon, S.-H.; Lee, I.-K.; Hyun, D.-H.; Chung, H.-Y.; Yoon, G. Sterol regulatory element-binding protein (SREBP)-1-mediated lipogenesis is involved in cell senescence. Journal of Biological Chemistry 285:29069-29077; 2010.

[47] Furukawa, S.; Fujita, T.; Shimabukuro, M.; Iwaki, M.; Yamada, Y.; Nakajima, Y.; Nakayama, O.; Makishima, M.; Matsuda, M.; Shimomura, I. Increased oxidative stress in obesity and its impact on metabolic syndrome. Journal of Clinical Investigation 114:1752; 2004.

[48] Gorgani-Firuzjaee, S.; Adeli, K.; Meshkani, R. Inhibition of SH2-domain-containing inositol 5-phosphatase (SHIP2) ameliorates palmitate induced-apoptosis through regulating Akt/FOXO1 pathway and ROS production in HepG2 cells. Biochemical and Biophysical Research Communications 464:441-446; 2015.

[49] Zhang, S.-L.; Chen, Y.-W.; Tran, S.; Chenier, I.; Hebert, M.-J.; Ingelfinger, J. R. Reactive oxygen species in the presence of high glucose alter ureteric bud morphogenesis. Journal of the American Society of Nephrology 18:2105$2115 ; 2007$.

[50] Mustapha, N. M.; Tarr, J. M.; Kohner, E. M.; Chibber, R. NADPH oxidase versus mitochondria-derived ROS in glucose-induced apoptosis of pericytes in early diabetic retinopathy. Journal of ophthalmology 2010; 2010.

[51] Gautier-Stein, A.; Soty, M.; Chilloux, J.; Zitoun, C.; Rajas, F.; Mithieux, G. Glucotoxicity induces glucose-6phosphatase catalytic unit expression by acting on the interaction of HIF-1 $\alpha$ with CREB-binding protein. Diabetes 61:2451-2460; 2012.

[52] Xie, J.; Onnockx, S.; Vandenbroere, I.; Degraef, C.; Erneux, C.; Pirson, I. The docking properties of SHIP2 influence both JIP1 tyrosine phosphorylation and JNK activity. Cellular signalling 20:1432-1441; 2008.

[53] Bakan, I.; Laplante, M. Connecting mTORC1 signaling to SREBP-1 activation. Current opinion in lipidology 23:226$234 ; 2012$.

[54] Lamming, D. W.; Sabatini, D. M. A Central role for mTOR in lipid homeostasis. Cell metabolism 18:465-469; 2013.

[55] Düvel, K.; Yecies, J. L.; Menon, S.; Raman, P.; Lipovsky, A. I.; Souza, A. L.; Triantafellow, E.; Ma, Q.; Gorski, R.; Cleaver, S. Activation of a metabolic gene regulatory network downstream of mTOR complex 1. Molecular cell 39:171$183 ; 2010$.

[56] Memmott, R. M.; Dennis, P. A. Akt-dependent and-independent mechanisms of mTOR regulation in cancer. Cellular signalling 21:656-664; 2009.

[57] Inoki, K.; Zhu, T.; Guan, K.-L. TSC2 mediates cellular energy response to control cell growth and survival. Cell 115:577-590; 2003. 
[58] Choudhari, S. R.; Khan, M. A.; Harris, G.; Picker, D.; Jacob, G. S.; Block, T.; Shailubhai, K. Deactivation of Akt and STAT3 signaling promotes apoptosis, inhibits proliferation, and enhances the sensitivity of hepatocellular carcinoma cells to an anticancer agent, Atiprimod. Molecular cancer therapeutics 6:112-121; 2007.

[59] Zheng, L.; Yang, W.; Wu, F.; Wang, C.; Yu, L.; Tang, L.; Qiu, B.; Li, Y.; Guo, L.; Wu, M. Prognostic significance of AMPK activation and therapeutic effects of metformin in hepatocellular carcinoma. Clinical Cancer Research 19:53725380; 2013. 


\section{Figure Legends:}

\section{Figure 1}

Effect of high glucose on SHIP2 expression at mRNA and protein levels. HepG2 cells were treated with $33 \mathrm{mM}$ glucose for $24 \mathrm{~h}$ and then the expression of SHIP2 at mRNA (A) and protein levels (B) were analyzed using the real-time PCR and western blotting, respectively. Represented data are from three independent experiments and are means \pm SEM. HG, high glucose $(33 \mathrm{mM}) ; \mathrm{NG}$, normal glucose $(5.5 \mathrm{mM}) .{ }^{*}: \mathrm{p}<0.0001$

\section{Figure 2}

Importance of SHIP2 inhibition in high glucose-induced lipogenesis. A-B):Generation of stable HepG2 cells. HepG2 stable cells were generated by infecting the cells with the viral supernatants followed by stable selection with $2 \mu \mathrm{g} / \mathrm{ml}$ puromycin. Real-time PCR and western blotting were used to detect SHIP2 mRNA and protein levels in HepG2 stable cells. A: Protein levels of SHIP2 in HepG2 stable cells. B) Analysis of Akt phosphorylation for assessment of the SHIP2 functionality.C-I): The influence of SHIP2 inhibition on high glucose-induced lipogenesis and the secretion of apoB. HepG2 cells and the cells expressing the SHIP2-DN gene were used in the absence or presence of high glucose $(33 \mathrm{mM})$ for $24 \mathrm{~h}$. C-D:Qualitative and quantities analyses of lipogenesis by oil red o staining, E-F: the amount of intra and extracellular TG levels, respectively, G:apoB secretion, $\mathrm{H}$ and I: the expression of the lipogenic genes such as MTP and ACC, K-M: the changes of SREBP1c protein and phosphorylation of ACC and FOXO1. Represented data are from three independent experiments and are means \pm SEM.DN: overexpresseddominant negative SHIP2 gene, WT: overexpressed wild typeSHIP2 gene, NG: normal glucose (5.5mM), HG: High glucose (33mM), BSA: Bovine Serum Albumin, Ins: insulin $100 \mathrm{nM}, \mathrm{TG}$ : triglyceride, *: $\mathrm{p}<0.0001$

\section{Figure 3}

Effect SHIP2 modulation on ROS production. HepG2 cells and the cells expressing GFP as control and DN and WT SHIP2 genes were used in these experiments. A: oil red o staining after treatment of the cells with 50 $\mu \mathrm{M}$ ascorbic acid [34], as ROS scavenger, B-E: the expression of the key lipogenic genes such as SREBP1c, ACC, FAS and MTP at the presence of ascorbic acid, E): apoB secretion at the presence of ascorbic acid in SHIP2 modulated cells. G: ROS level in the SHIP2 modulated cells using DHE probe. H): Mitochondrial ROS level in SHIP2 modulated cells using MitoSOX red dye. I and L): Total lipid (oil red o staining) and TG content 
at the presence of NADPH oxidase inhibitor (DPI $10 \mu \mathrm{M}$ ). K and M): Total lipid (oil red o staining) and TG content at the presence of ETC inhibitor (rotenone $1 \mu \mathrm{M}$ ). Represented data are from three independent experiments and are means \pm SEM. DN: overexpresseddominant negative SHIP2 gene, WT: overexpressed wild typeSHIP2 gene, Asc: ascorbic acid $(50 \mu \mathrm{M})$, NG: normal glucose $(5.5 \mathrm{mM})$, HG: High glucose $(33 \mathrm{mM})$, R: rotenone, DPI: Diphenylene iodonium *: $\mathrm{p}<0.0001$

\section{Figure 4}

The effect of SHIP2 inhibition on MAPK pathway in high glucose condition.HepG2 cells and the cells expressing the GFP as control and DN and WT genes were used in the absence or presence of high glucose (33mM) for 24h.A): total lipid content at the presence of MAPKs pathway inhibitors (JNK, P38 and ERK), B: the expression of the key lipogenic genes at the presence of JNK inhibitors, C: apoB100 secretion at the presence of JNK inhibitors, D: MAPKs protein phosphorylation in SHIP2 modulated cells, E: Effect of SHIP2 inhibition on high glucose-induced JNK phosphorylation at the presence anisomycin $(50 \mu \mathrm{M})$, F:total lipid content at the presence anisomycin $(50 \mu \mathrm{M})$ as JNK activator, G: apoB100 secretion at the presence of anisomycin $(50 \mu \mathrm{M})$. H:The expression of MTP gene at the presence of anisomycin $(50 \mu \mathrm{M})$, I: JNK phosphorylation inthe SHIP2-WT and GFP control cells at the presence of ascorbic acid (50 $\mu \mathrm{M})$, K): phosphoAkt level at the presence of high glucose and JNK inhibitor in the GFP and SHIP2-WTcells, L): phospho-Akt level in the GFP and SHIP2-DN cells at the presence of anisomycin, M): the phosphorylation changes in SHIP2 modulated cells. Represented data are from three independent experiments and are means \pm SEM. DN: overexpresseddominant negative SHIP2 gene, WT: overexpressed wild typeSHIP2 gene, NG: normal glucose (5.5mM), HG: high glucose $(33 \mathrm{mM})$, Ins: insulin $100 \mathrm{nM}$, In: inhibitors, JNK In: inhibitor of JNK, A: Anisomycin $(50 \mu \mathrm{M}), *: \mathrm{P}<0.001$

\section{Figure5}

\section{The effectsSHIP2 inhibition on the AMPK-mTOR-SREBP1c pathway in the high glucose condition.} HepG2 cells and the cells expressing GFP as control and SHIP2-DN and SHIP2-WT genes were used in the absence or presence of high glucose $(33 \mathrm{mM})$ for $24 \mathrm{~h}, \mathrm{~A}$ : the AMPK phosphorylation in SHIP2 modulated cells, B: the mTOR ser2481 phosphorylation in the cells treated with high glucose, C: total lipid content at the presence of rapamycin $(20 \mathrm{nM})$ and high glucose, D: TG level of the cells at the presence of rapamycin $(20 \mathrm{nM})$ and high glucose, E) total lipid content at the presence of metformin $(5 \mathrm{mM})$ and high glucose, F: TG level of the cells at the presence of metformin $(5 \mathrm{mM})$ and high glucose,G: phospho- AMPK, phospho-mTOR, SREBP1c and phospho-ACC levels at the presence of high glucoseand rapamycin $(20 \mathrm{nM}), \mathrm{I})$ phospho- AMPK, 
phospho-mTOR, SREBP1c and phospho-ACC levels at the presence of high glucoseand metformin $(5 \mathrm{mM})$. Represented data are from three independent experiments and are means \pm SD. DN: overexpressed dominant negative SHIP2 gene, WT: overexpressed wild type SHIP2 gene, Normal glucose (5.5mM), HG: High glucose $(33 \mathrm{mM}),{ }^{*}: \mathrm{P}<0.001$

\section{Figure 6}

Fig 6: Molecular mechanisms linking SHIP2 to hepatic lipid homeostasis in the state of high glucose.High glucose appears to induce the de-novo lipogenesis and the secretion of apoB containing lipoproteins in HepG2 cells by affecting two signaling pathways including the ROS/JNK/Akt and the AMPK/mTROC1/SREBP1. Inhibition of SHIP2 expression could ameliorate high glucose-induced secretion of apoB containing lipoproteins by an ROS/JNK/Akt-dependent mechanism. SHIP2 inhibition could also prevent high glucoseinduced de-novo lipogenesis by an Akt-independent mechanism involving the AMPK/mTOR/SREBP1c signaling pathway. Altogether, a decreased de-novo lipogensis leading to a lower TG content along with a decreased expression of MTP resultsina reduced secretion of apoB containing lipoproteins (VLDL) in SHIP2DN cells. 
Fig 1
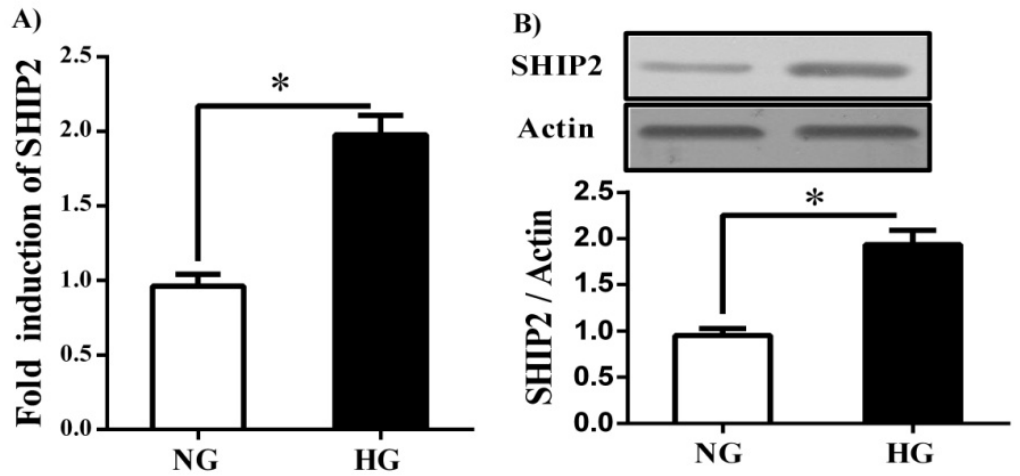
Fig 2:
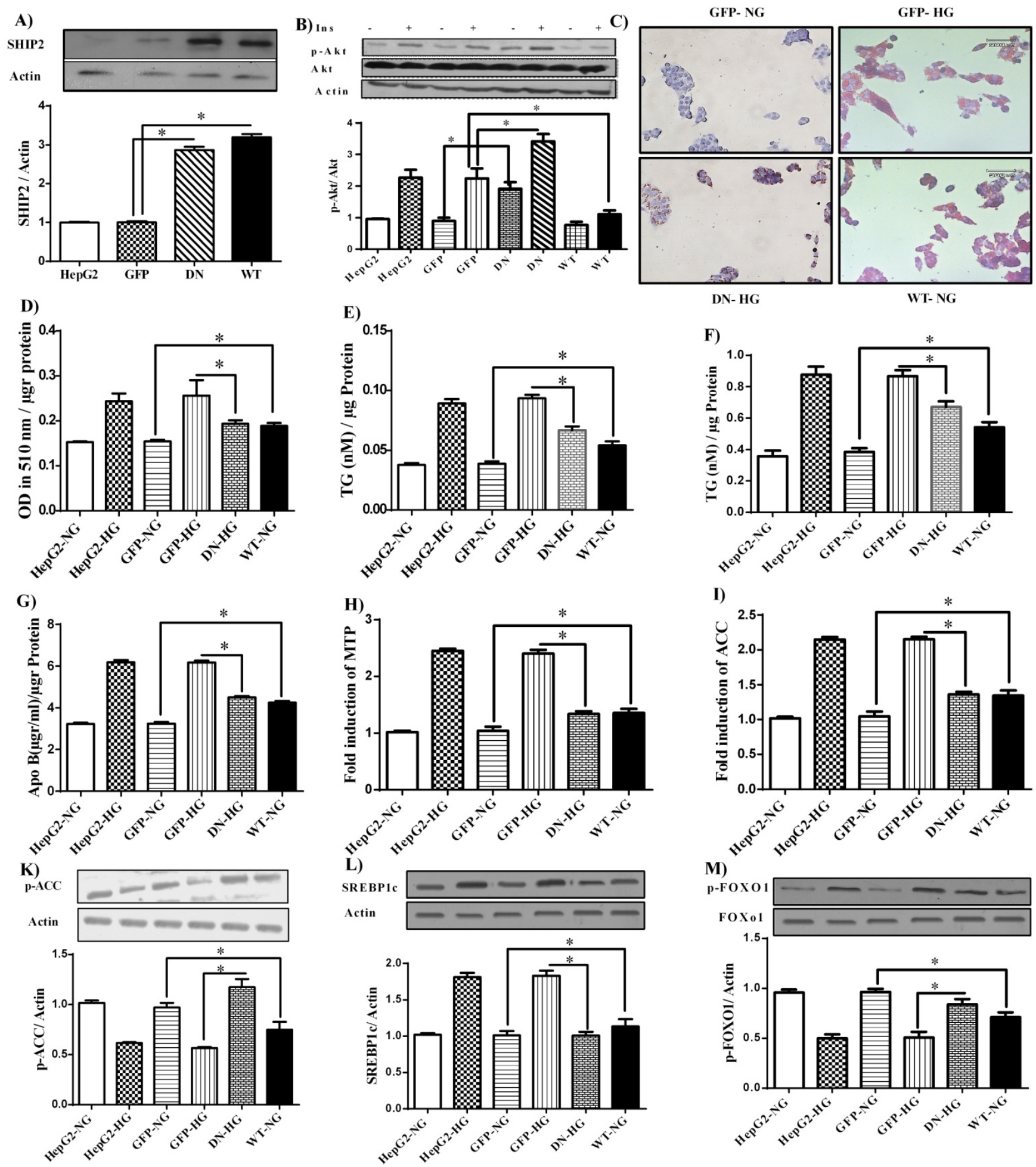
Fig 3:

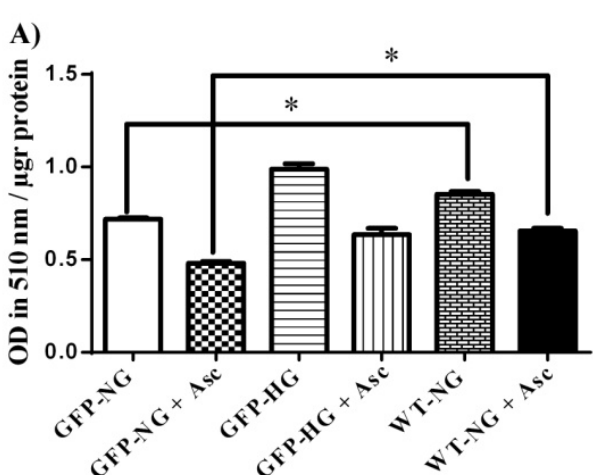

D)

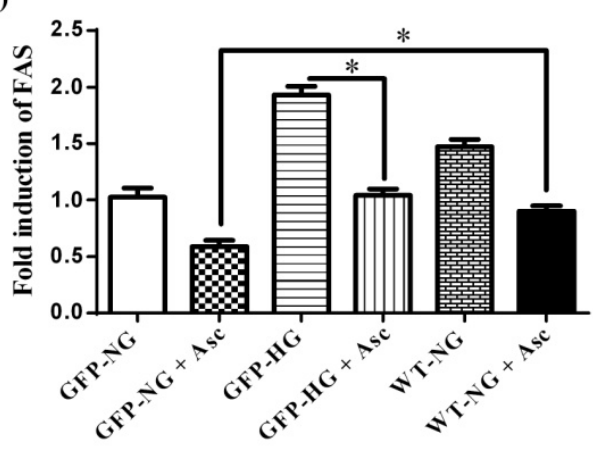

G)

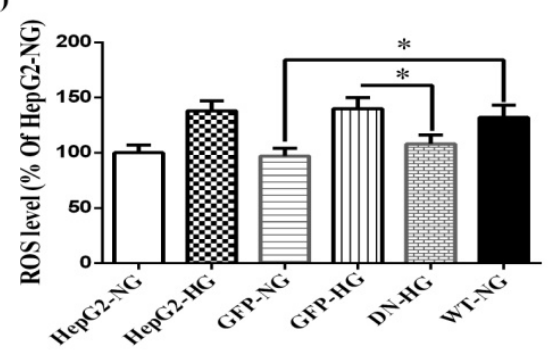

H)

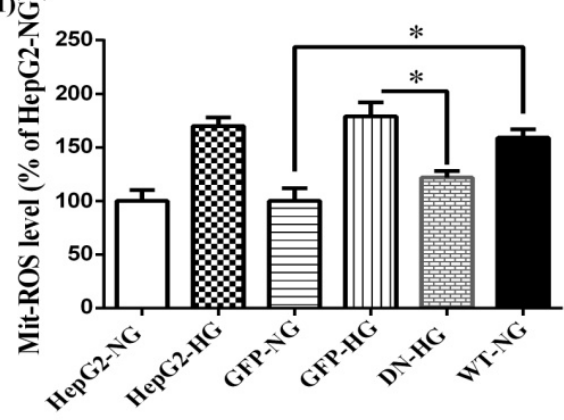

B)

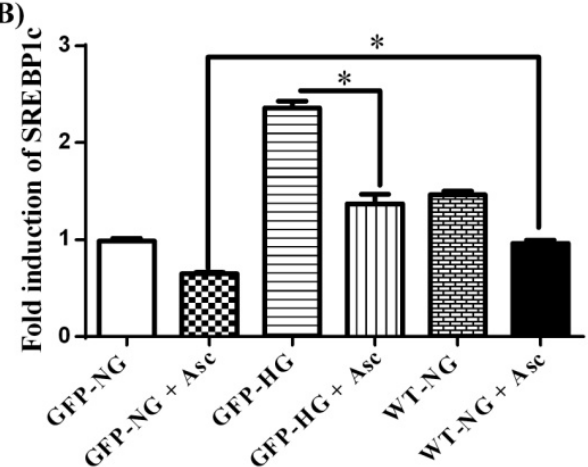

E)

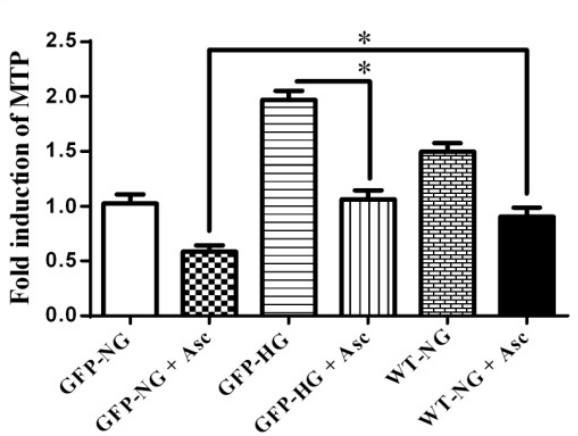

I)

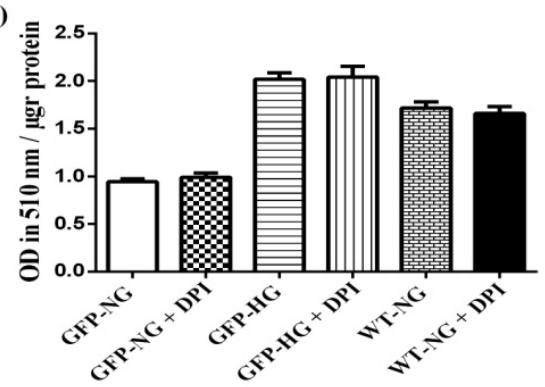

K)

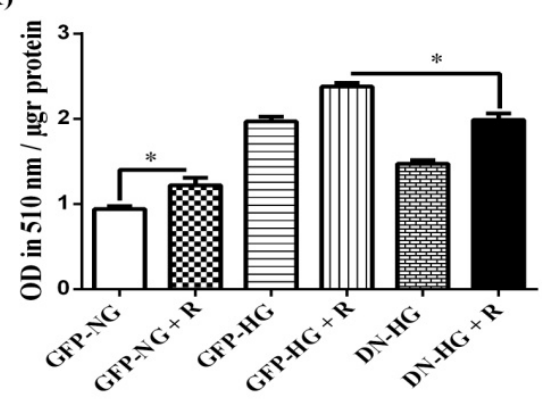

C)

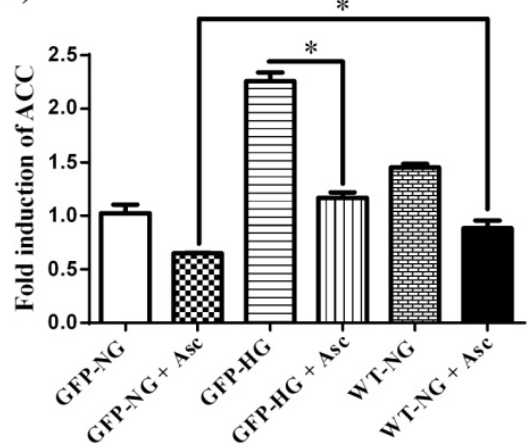

F)
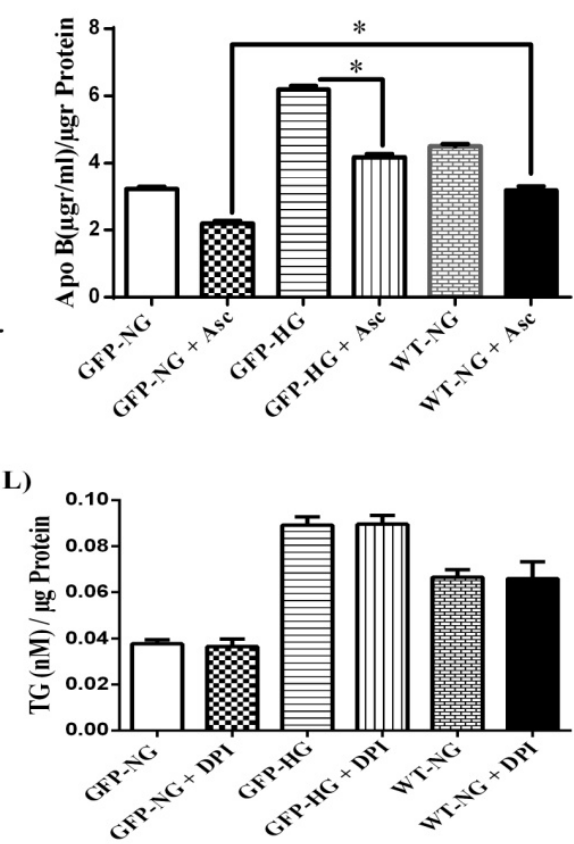

M)

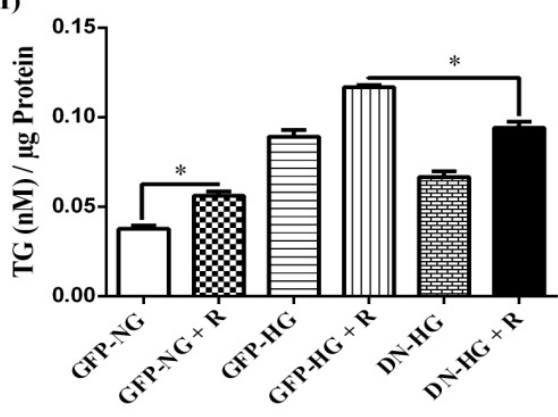


Fig 4:
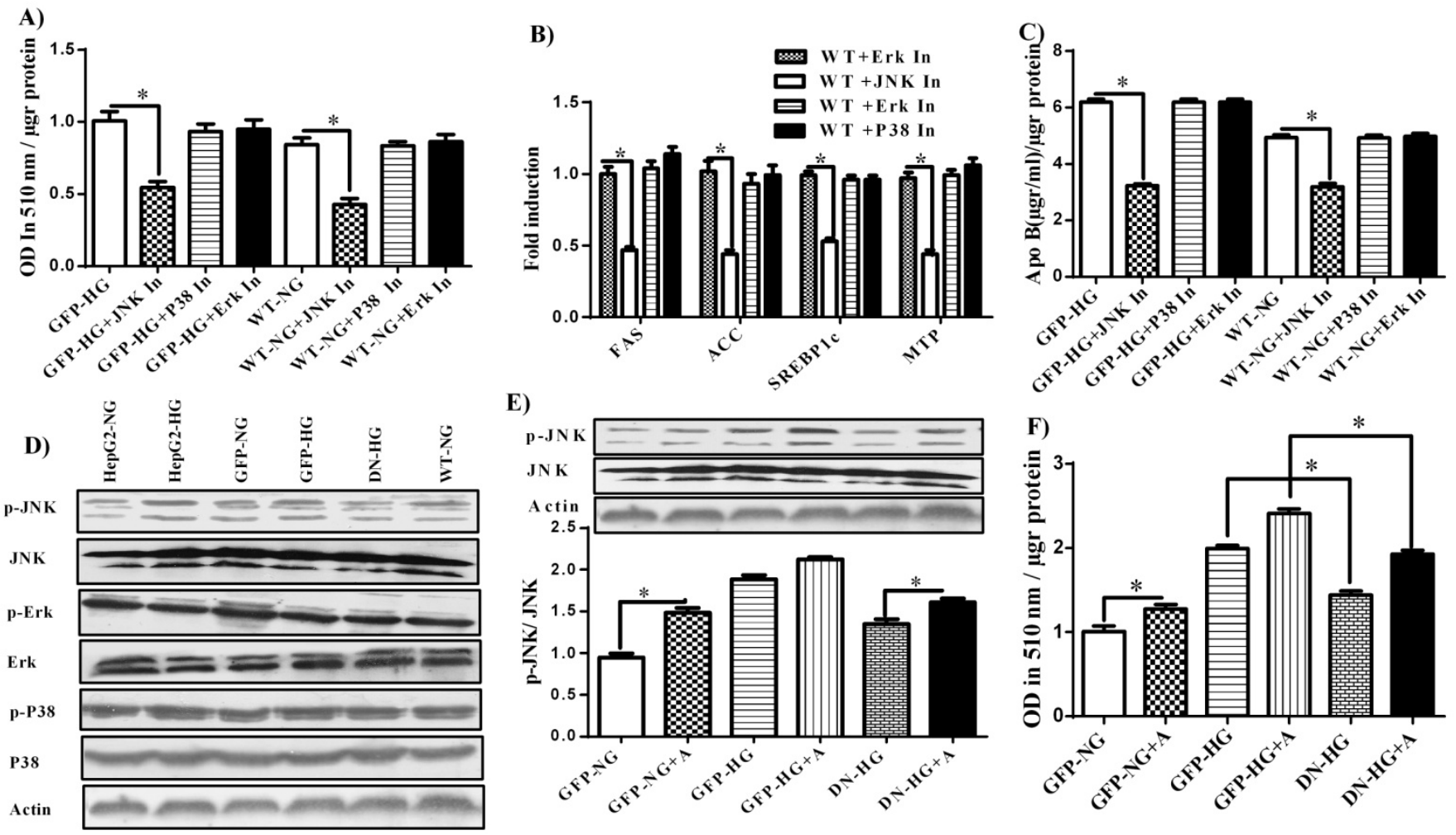

E)
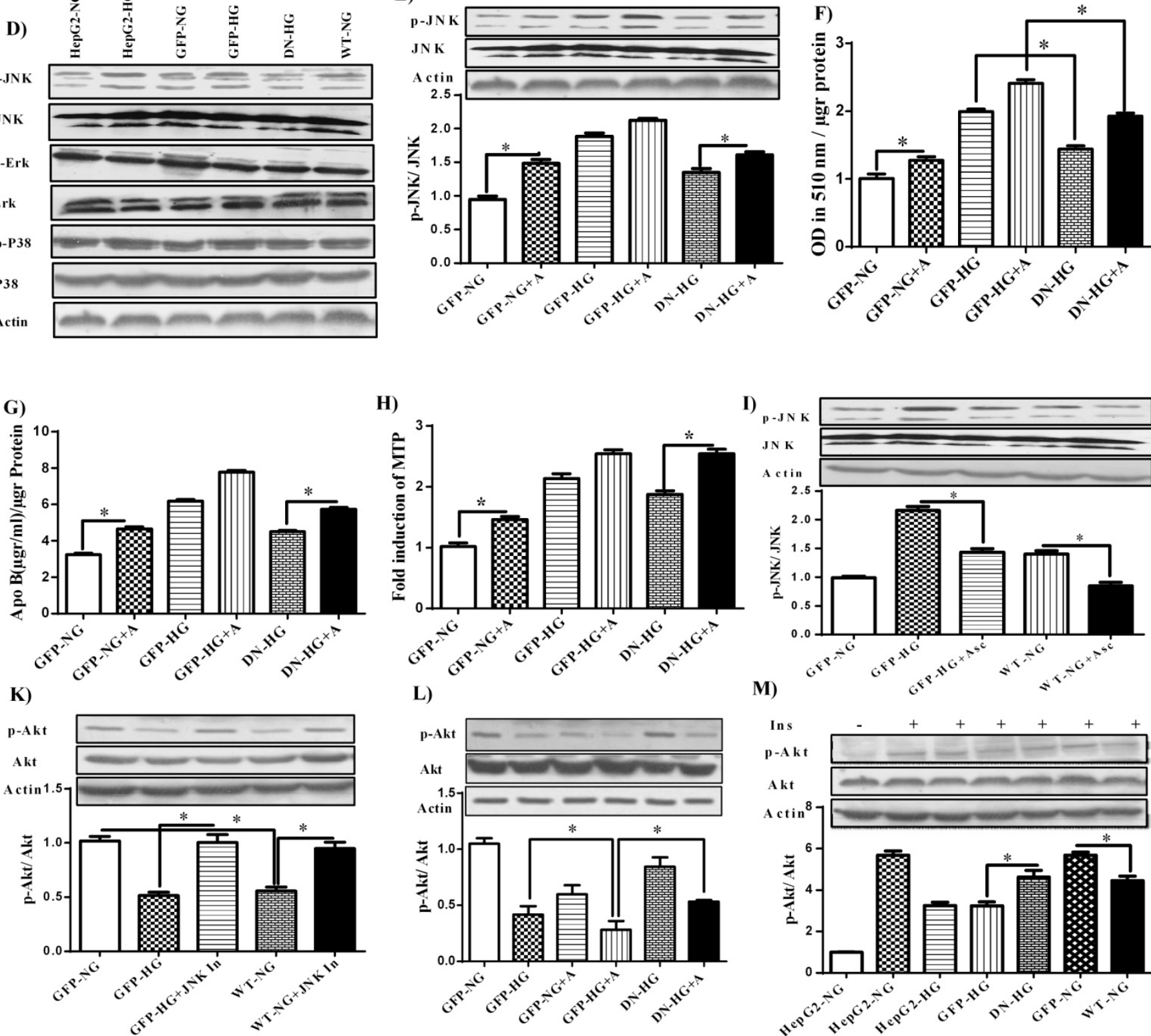

L)

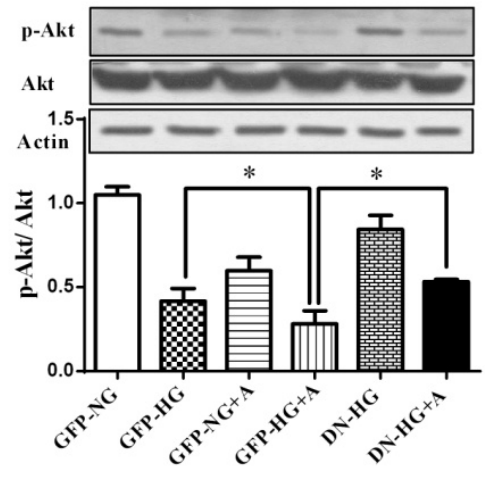

M)
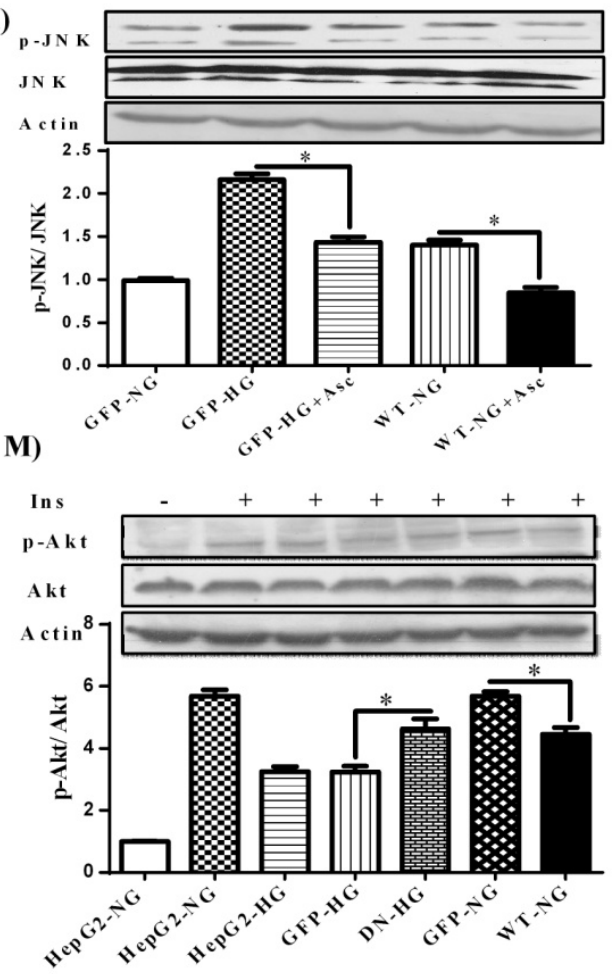
Fig 5:

A)

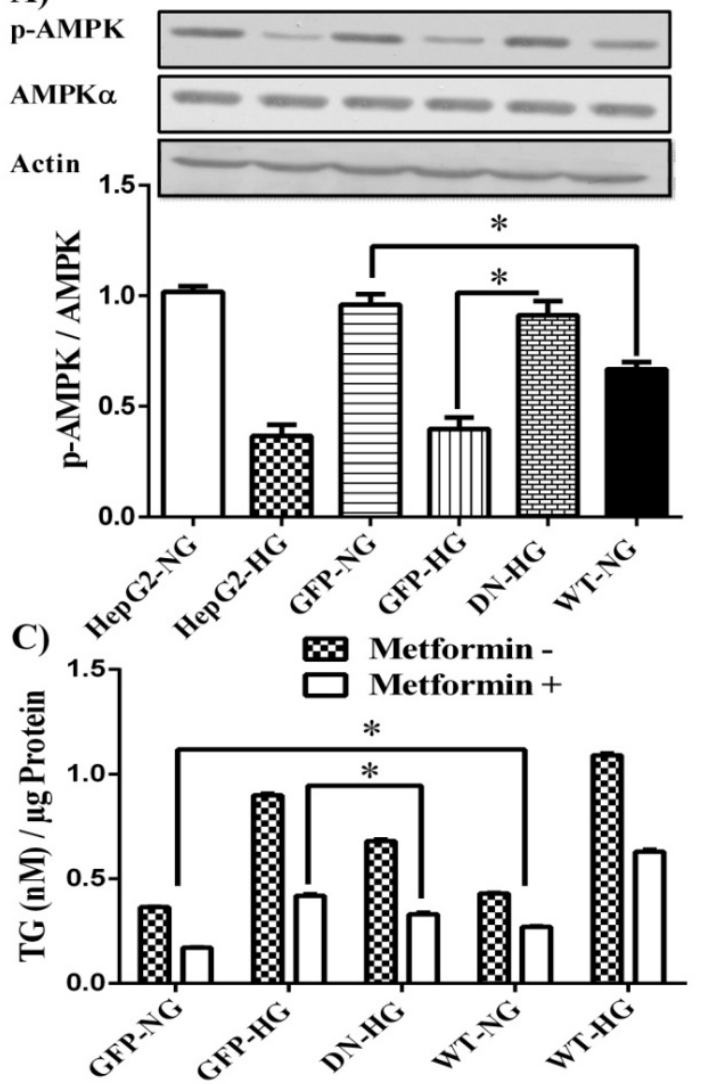

E)

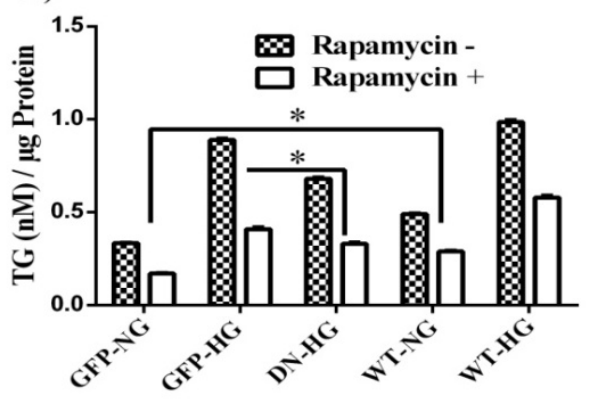

G)
B)

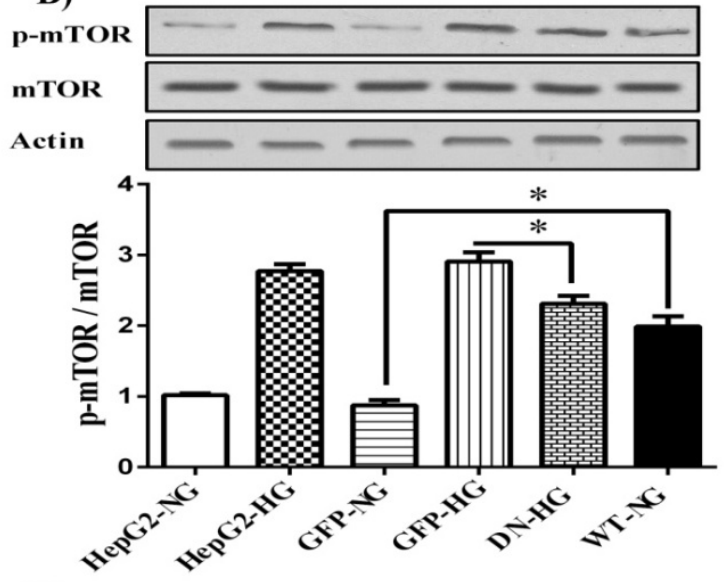

D)

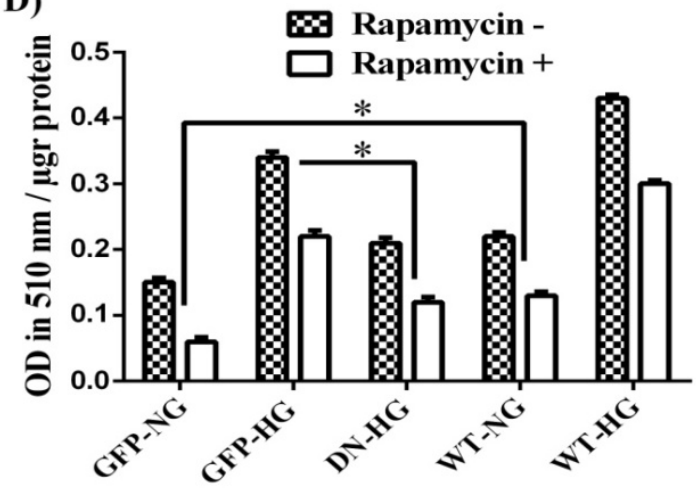

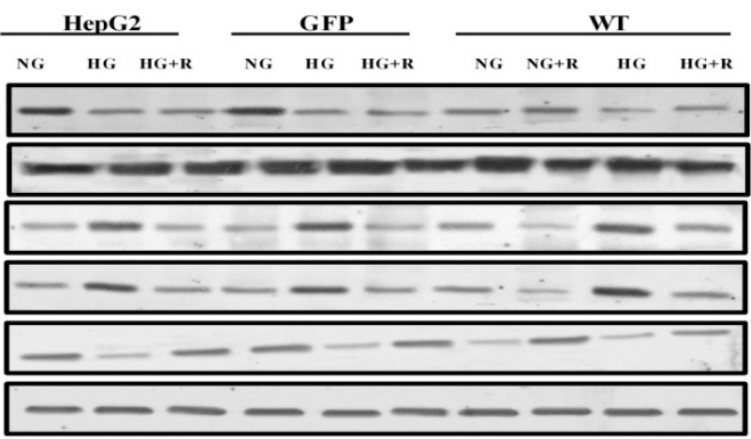

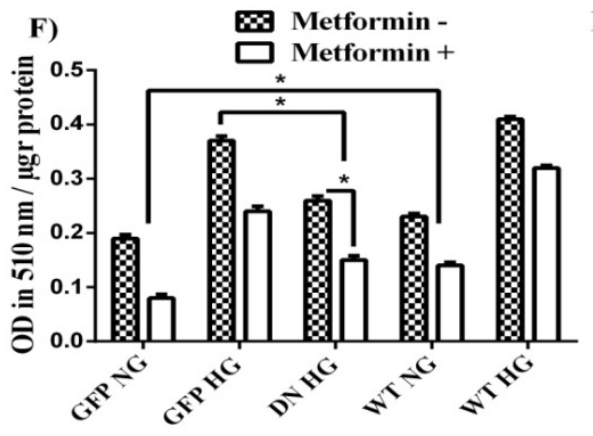

H)

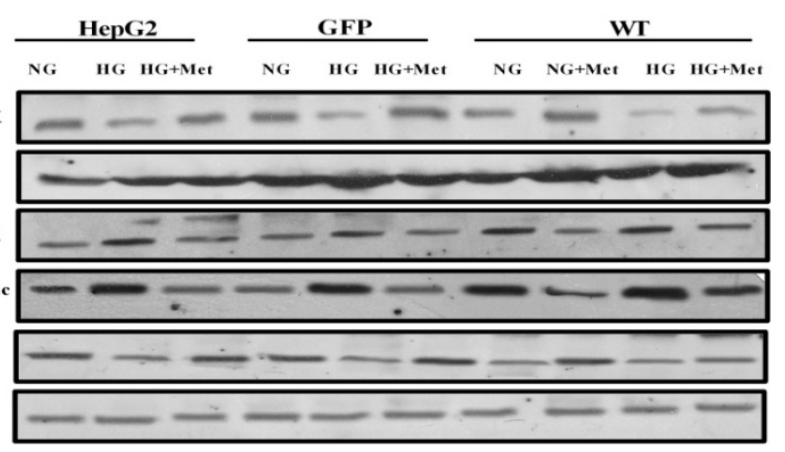


Fig 6:

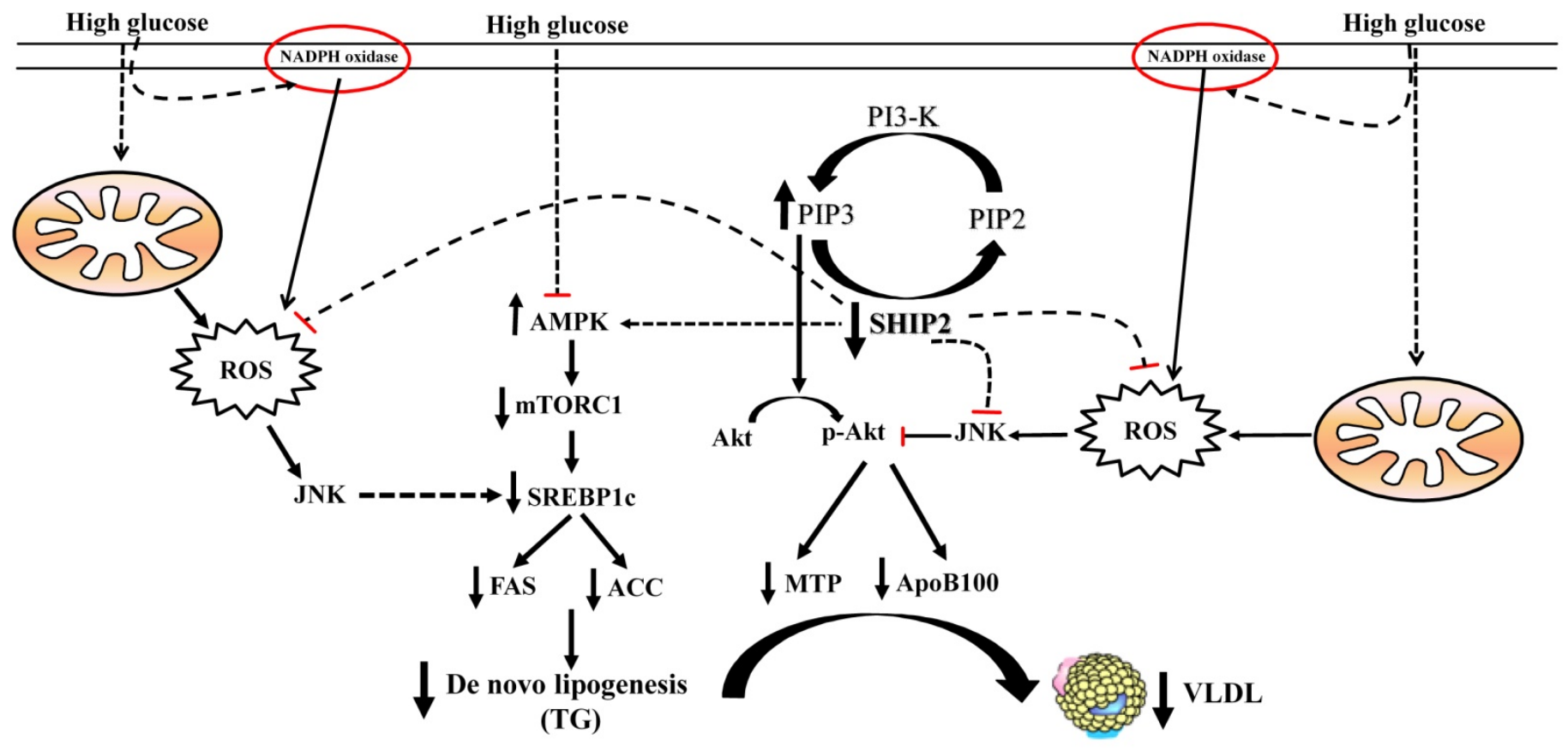

